\title{
32 Premio José Félix Patiño Restrepo Reinventar la presentación de trabajos de investigación
}

\author{
32th José Félix Patiño Restrepo Award \\ Reinvent the presentation of the research posters \\ $32^{\circ}$ Prêmio José Félix Patiño Restrepo \\ Reinventar a apresentação de trabalhos de investigação \\ Yadira Cortés Sanabria' \\ https://doi.org/10.35454/rncm.v4n3.300
}

La pandemia mundial por la enfermedad por coronavirus (COVID-19) ha llevado a enfrentar nuevos retos tanto en lo personal como en lo profesional; de manera que la Asociación Colombiana de Nutrición Clínica (ACNC) se ajustó a esta situación, y no solo cambió la modalidad a virtual del 35 Congreso sobre Avances en Metabolismo y Nutrición Clínica, sino de la presentación de los trabajos de investigación.

Los trabajos aceptados se presentaron en forma de póster, acompañados de una grabación que explica los puntos que cada investigador deseaba resaltar, lo cual permitió que estos se visitaran en cualquier momento durante el desarrollo del Congreso.

Este año, el Premio José Félix Patiño Restrepo llega a su versión 32 , con la participación de 47 trabajos, de los cuales 27 se presentaron en la modalidad de trabajos libres, 9 en trabajos de grado y 11 en protocolo de investigación. Para la evaluación de los trabajos se nombraron 22 pares académicos (15 nacionales y 7 internacionales: Chile, Perú, Paraguay, Costa Rica, México, Uruguay y Venezuela), quienes mediante un formato estandarizado evaluaron la calidad de cada trabajo, teniendo en cuenta los siguientes criterios en trabajos libres y trabajos de grado:

- Importancia de la pregunta de investigación

- Metodología realizada

- Interpretación de los resultados

- Presentación del documento.

\footnotetext{
Coordinadora de la Asociación Colombiana de Nutrición Clínica.
}

Protocolos:

- Importancia de la pregunta de investigación

- Metodología propuesta

- Planteamiento de análisis de resultados y presupuesto

- Presentación del documento.

Los ganadores son los siguientes trabajos:

\section{MODALIDAD: PROTOCOLO DE INVESTIGACIÓN}

- Premio: $\$ 2500000$ pesos colombianos

- Asociación entre el estado nutricional y el desempeño cognitivo en un grupo de adultos mayores del modelo de atención integral SerMás, IPS Universitaria

- Presentado por Mateo Londoño Pereira

- Escuela de Nutrición y Dietética, Universidad de Antioquia, Medellín.

\section{MODALIDAD:TRABAJO DE GRADO}

\section{Primer puesto}

- Premio: $\$ 2000000$ pesos colombianos

- Calidad nutricional de la dieta a partir del indicador de diversidad alimentaria MDD-W e índices de adecuación de micronutrientes en mujeres en edad fértil: estudio ELANS, Colombia 
- Presentado por María José Pedraza Serrano, Luz Nayibe Vargas Mesa, Georgina Gómez Salas

- Pontificia Universidad Javeriana, Bogotá.

\section{Segundo puesto}

- Premio: $\$ 500000$ pesos

- Elementos traza, hipotiroidismo primario y subclínico en el ciclo vital humano

- Presentado por Paula Sofia Guatibonza Vargas, Idana Valentina Torres Navarro, Juan Pablo Pineda Casadiego, José Manuel Oviedo Nieto

- Facultad de Medicina, Universidad El Bosque, Bogotá.

\section{MODALIDAD: TRABAJO LIBRE}

\section{Primer puesto}

- Premio: \$ 7000000

- Variación de parámetros ultrasonográficos en pacientes críticamente enfermos con SARS-CoV-2, COVID-19

- Presentado por Johan Sebastián Torres Mora, Ricardo Alfonso Merchán Chaverra, Yeny Marjorie Cuellar Fernández, Jorge Alexis Medina Parra

- Clínica Infantil Santa María del Lago y Clínica Colsanitas; Grupo de Investigación en Nutrición Clínica, Fundación Universitaria Sanitas (UNISANITAS), Bogotá.

\section{Segundo puesto}

- Premio: \$2000 000 cada uno

- Asociación entre la actividad y la condición física con masa corporal magra en niños españoles: estudio CALINA

- Presentado por Diana Paola Córdoba Rodríguez, Iris Iglesia, Alejandro Gómez Bruton, María L. Miguel Berges, Paloma Flores Barrantes, José Antonio Casajús, Luis A. Moreno, Gerardo Rodríguez Martínez

- Grupo de Alimentos Nutrición y Salud, Facultad de Ciencias, Pontificia Universidad Javeriana; Grupo de Investigación Crecimiento, Ejercicio, Nutrición y Desarrollo (GENUD), Universidad de Zaragoza, España.

\section{Segundo puesto}

- Doble carga nutricional en hogares colombianos: una aproximación desde los determinantes sociales en 2010

- Presentado por Yenny Paola Rueda Guevara, Catalina González Uribe

- Fundación Santa Fe de Bogotá y Universidad de los Andes, Bogotá.

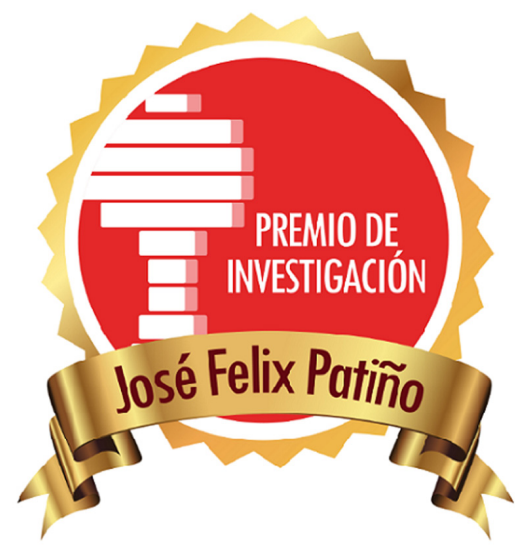




\title{
Trabajos de grado
}

\author{
Abstract ID: 158
}

\section{REVISIÓN DE REVISIONES: ELEMENTOS TRAZA, HIPOTIROIDISMO PRIMARIO Y SUBCLÍNICO EN EL CICLO VITAL HUMANO}

\author{
OVERVIEW OF REVIEWS: TRACE ELEMENTS, PRIMARY AND SUBCLINICAL HYPOTHYROIDISM IN THE HUMAN LIFE CYCLE
}

Idana Valentina Torres Navarro', Paula Sofía Guatibonza Vargas', José Manuel Oviedo Nieto', Juan Pablo Pineda Casadiego', Gustavo Alfonso Díaz Muñoz².

Introducción: El hipotiroidismo es una enfermedad con alta prevalencia a nivel mundial, por su parte los oligoelementos tienen un papel importante en la síntesis y metabolismo de hormonas tiroideas, por lo cual una ingesta inadecuada se encuentra directamente relacionada con la aparición de esta enfermedad. La información sobre su relación es abundante dificultando su búsqueda, por lo que realizamos una síntesis de revisiones sistemáticas y metaanálisis para facilitar su acceso.

Objetivo: Describir la relación de los oligoelementos con la prevención y tratamiento del hipotiroidismo primario y subclínico en las etapas del ciclo vital humano.

Materiales y métodos: Revisión de revisiones que involucró revisiones sistemáticas y metaanálisis en PubMed, BVS, Embase, Cochrane, Scielo y SCOPUS

${ }^{1}$ Facultad de Medicina, Universidad El Bosque. Bogotá, Colombia. ${ }^{2}$ Instituto de Investigación en Nutrición, Genética y Metabolismo, Facultad de Medicina, Universidad El Bosque. Bogotá, Colombia. hasta 17 junio 2020. La selección y extracción se realizó por duplicado. La calidad se evaluó mediante AMSTARD-2.

Resultados: se encontraron 940 artículos y 10 se incluyeron para la revisión. 7 revisiones incluyeron únicamente experimentos y 7 contaron con calidad alta. La suplementación de yodo tiene efectos bilaterales, se relaciona con aumento o disminución de la TSH. Así mismo, la suplementación con yodo incrementa su excreción urinaria y disminuye la tiroglobulina, sin embargo, estos hallazgos cambian según el grupo de edad y la dosis. La suplementación con selenio solo demostró efectividad en la reducción de TPOAb y TgAb al emplearse con levotiroxina.

Conclusiones: Solo se encontró información sobre la suplementación con selenio y yodo asociado con las hormonas tiroideas, siendo estos dependientes del ciclo vital estudiado.

Palabras clave: hipotiroidismo primario, hipotiroidismo subclínico, oligoelementos, yodo, selenio.

Keywords: Primary Hypothyroidism; Subclinical Hypothyroidism; Trace Elements; lodine; Selenium.

\section{Abstract ID: 163}

\section{DIETAS BASADAS EN PLANTAS Y SU RELACIÓN CON EL RIESGO Y/O DESENLACE CARDIOVASCULAR EN ADULTOS Y ADULTOS MAYORES}

\section{PLANT-BASED DIETS AND THEIR RELATIONSHIP WITH CARDIOVASCULAR RISK AND/OR DISENGAGEMENT IN ADULTS AND OLDER ADULTS}

Johan Andrea Mojica Matallana'

Introducción: La Enfermedad cardiovascular es la principal causa de mortalidad en el mundo, la alimentación como parte fundamental de su prevención y tratamiento ha sido ampliamente estudiada en los últimos años.

Objetivo: Describir la relación entre el cumplimiento de patrones alimentarios de dietas basadas en plantas y el riesgo y/o desenlace cardiovascular en adultos y adultos mayores.

Método: La búsqueda bibliográfica y desarrollo de la revisión se llevó a cabo teniendo en cuenta la guía PRISMA 2009, las bases de datos utilizadas para la búsqueda fueron Pubmed, Science Direct y EBSCOhost. Se incluyeron estudios de cohorte prospectivos desde 2015 a la fecha, en adultos y adultos mayores en los cuales se caracterizarán los patrones de alimentación mediante un índice de Dieta basada en plantas y se relacionara con enfermedad cardiovascular.

'Universidad de Chile, Chile.
Resultados: De un total de 18 artículos que cumplían con los principales criterios de inclusión se seleccionaron 5 por la metodología de investigación utilizada y su pertinencia. Todos los estudios seleccionados mostraron que una mayor adherencia o cumplimiento de patrones de PBD (aquellos individuos ubicados en los quintiles mayores) tienen un efecto protector sobre la ECV y su mortalidad asociada.

Conclusiones: La adherencia a una alimentación basada principalmente en plantas pero sin la exclusión completa de alimentos de origen animal, puede tener beneficios sobre el riesgo y desenlace de ECV. Las PBD también tienen efectos positivos en el medio ambiente, otro motivo para incentivar su aplicación y consumo.

Palabras clave: adulto mayor, adulto, dietas basadas en plantas, enfermedad cardiovascular, riesgo cardiovascular.

Keywords: Adult; Cardiovascular Disease; Cardiovascular Risk; Erderly; Plant Based Diet. 
Abstract ID: 167

\title{
CARACTERÍSTICAS ALIMENTARIAS EN UN GRUPO PEDIÁTRICO CON AUTISMO DE 2 A 18 AÑOS EN PAÍSES DEL CONTINENTE AMERICANO
}

\author{
DIETARY CHARACTERISTICS IN A PEDIATRIC GROUP WITH AUTISM FROM 2 TO 18 YEARS OLD IN COUNTRIES OF THE AMERICAN \\ CONTINENT
}

Valentina Marysol Cuevas Cuevas'

Introducción: El autismo es un trastorno estático del desarrollo neurológico que persiste toda la vida y que incluye un amplio margen de alteraciones conductuales. En el presente estudio se analizan las características de la población estudiada desde el enfoque de alimentación.

Objetivo: describir las características alimentarias en 96 individuos con Autismo de grupo pediátrico según las respuestas de los familiares encuestados de Febrero a Marzo del 2021.

Metodología: estudio observacional y descriptivo con enfoque de tipo corte transversal. Se incluyeron a 96 familiares y/o padres de niños con Autismo entre los 2 a 18 años pertenecientes a grupos de educación y concientización por medio de redes sociales, en distintos países del continente americano. El período de recaudación de muestra fue de Febrero-Marzo del 2021

'Universidad Nacional Autónoma de Honduras. Tegucigalpa, Honduras.
Resultados: el $23 \%$ de la población son del sexo femenino y el $77 \%$ del sexo masculino. El $62.5 \%$ tiene entre 2 a 5 años de edad y el $32.2 \%$ de 6 a 18 años. Sobre las preferencias alimentarias limitadas, el $68 \%$ si las presenta, en gran diferencia con el $32.2 \%$ que no. El $73 \%$ nunca llevó a consulta nutricional, y apenas el $23 \%$ sí.

Conclusión: En TEA es fundamental identificar las características médicas, psicológicas y/o psiquiátricas para lograr un abordaje nutricional completo. El Nutricionista tiene la responsabilidad de educar, capacitar y ofrecer soluciones de una alimentación variada, poco restrictiva y que cumpla con las características adecuadas pese a sensibilidades o problemas sensoriales del niño, para lograr un adecuado estado nutricional.

Palabras clave: nutrición, autismo, alimentación.

Keywords: Nutrition; Autism; Feeding.

Abstract ID: 172

\section{SOPORTE NUTRICIONAL EN PACIENTE PEDIÁTRICO EN LA UNIDAD DE CUIDADOS INTENSIVOS: PROPUESTA DE PROTOCOLO DE ACCIÓN}

\author{
NUTRITIONAL SUPPORT IN PEDIATRIC PATIENTS IN THE INTENSIVE CARE UNIT: PROPOSED ACTION PROTOCOL
}

María José Criales Saavedra'

Introducción: El deterioro del estado nutricional y la desnutrición son comunes en pacientes pediátricos críticamente enfermos en unidad de cuidados intensivos pediátricos (UCIP), su prevalencia oscila entre $2,9 \%$ y $31 \%$. Un aporte nutricional adecuado disminuye su riesgo de morbimortalidad, por esto necesario el desarrollo de instrumentos que establezcan medidas y acciones para detectar de forma eficiente condiciones que aumentan el deterioro del estado nutricional en este tipo de pacientes.

Objetivo: Revisar guías de práctica clínica y literatura científica en soporte nutricional para pacientes pediátricos entre los 1 y 12 años de edad que se encuentran en UCIP, que permita proponer protocolos de actuación para uso clínico.

Metodología: Basados en los lineamientos de Vallejo-Ortega et al. (2016), se definieron alcances, equipo desarrollador y las preguntas clínicas bajo la estrategia PICO. Se realizó una búsqueda de guías de práctica clínica y litera-

'Universidad Nacional de Colombia. Bogotá, Colombia. tura científica que fueron evaluadas por AGREE II y GRADE, respectivamente. Se establecieron las recomendaciones para cada pregunta $y$, finalmente, se discutió el protocolo con dos evaluadores para así incluir sus recomendaciones a la versión final.

Resultados: Se evaluaron 4 guías de práctica clínica y 26 artículos científicos con los que se elaboró una propuesta de protocolo desarrollada en 10 preguntas clínicas y en la que se resaltan hallazgos como: requerimientos, objetivo de ingesta proteica, antropometría, metabolismo de macronutrientes, soporte parenteral suplementario y principales complicaciones.

Conclusión: Esta propuesta de protocolo permitirá disminuir la variabilidad en la práctica clínica en UCIP y favorecerá una adecuada intervención nutricional en pacientes pediátricos críticos que requieran un soporte nutricional especializado.

Palabras clave: protocolo, soporte, nutricional, pediátrico, UCIP.

Keywords: Protocol; Support; Nutritional; Pediatric; PICU.

\section{Abstract ID: 173 \\ CALIDAD NUTRICIONAL DE LA DIETA A PARTIR DEL INDICADOR DE DIVERSIDAD ALIMENTARIA MDD-W E ÍNDICE DE ADECUACIÓN DE MICRONUTRIENTES EN MUJERES EN EDAD FÉRTIL: ESTUDIO ELANS-COLOMBIA}

\section{NUTRITIONAL QUALITY OF THE DIET BASED ON THE FOOD DIVERSITY INDICATOR MDD-W AND MICRONUTRIENT ADEQUACY INDEX IN WOMEN OF CHILDBEARING AGE: ELANS-COLOMBIA STUDY}

María José Pedraza Serrano', Luz Nayibe Vargas Mesa², Georgina Gómez Salas³.

Introducción: La calidad de la dieta, medida por la diversidad alimentaria y la adecuación de micronutrientes, se constituye como una herramienta clave para el monitoreo de una alimentación saludable, especialmente en mujeres en edad fértil (MEF), que son vulnerables a la deficiencia de vitaminas y minerales. El indicador MDD-W ha sido ampliamente utilizado en países de ingresos bajos y medios.
Objetivo: Este estudio, fundamentado en datos secundarios del estudio ELANS, tuvo como objetivo determinar la calidad nutricional de la dieta de las MEF en 11 ciudades de Colombia.

Métodos: La ingesta dietaria de 483 mujeres se evaluó a partir de un recordatorio de 24 horas de pasos múltiples. La diversidad alimentaria se calculó con base en diez grupos de alimentos, considerando un punto de corte de 
consumo $\geq 5$. Se utilizaron los Índices de Adecuación de Nutrientes (NAR) y su respectiva media (MAR) para determinar la adecuación de micronutrientes.

Resultados: La media del DDS fue de 4,72, siendo menor en la ciudad de Cali y en el nivel socioeconómico bajo. La vitamina $D$ y $A$ mostraron un NAR promedio de 0,43 y 0,52 , respectivamente. El grupo con una dieta diversa

'Facultad de Ciencias, Pontificia Universidad Javeriana. Bogotá D.C., Colombia. ${ }^{2}$ Departamento de Nutrición y Bioquímica, Facultad de Ciencias, Pontificia Universidad Javeriana. Bogotá D.C., Colombia.

${ }^{3}$ Departamento de Bioquímica, Escuela de Medicina, Universidad de Costa Rica. San José, Costa Rica.
(DDS $\geq 5$ ) informó un mayor consumo de grupos de alimentos saludables y menor de bebidas azucaradas.

Conclusión: La calidad nutricional de la dieta en las MEF colombianas difiere según factores sociodemográficos, pero no se encuentra muy lejos de ser óptima.

Palabras clave: calidad nutricional de la dieta, diversidad alimentaria, micronutrientes, adecuación de nutrientes, mujeres en edad fértil.

Keywords: Dietary Nutritional Quality; Dietary Diversity; Micronutrients; Nutrient Adequacy; Women of Childbearing Age.

Abstract ID: 178

\title{
CARACTERIZACIÓN DE LA COMPOSICIÓN CORPORAL POR CINCO COMPONENTES Y DEXA EN DEPORTISTAS DE 8 A 17 AÑOS DE MEDELLÍN
}

\author{
CHARACTERIZATION OF BODY COMPOSITION BY FIVE COMPONENTS AND DEXA IN ATHLETES FROM 8 TO 17 YEARS OLD IN MEDELLÍN
}

Maximiliano Kammerer López', Alejandra Sánchez Sanguino', Vanesa Castañeda Ramírez', Sara González Pajón', Jessica Alejandra Ramírez Acevedo'

Objetivo: Determinar la composición corporal utilizando Método de Cinco Componentes y Absorciometría Dual por Energía de Rayos X (DEXA) en deportistas de 8 a 17 años del Team Medellín.

Método: Enfoque cuantitativo de tipo observacional descriptivo-transversal, con direccionalidad retrospectiva obteniendo datos de fuente secundaria.

Estudio realizado con 100 deportistas de diferentes disciplinas deportivas. Para los análisis se utilizaron estadísticas descriptivas, análisis bivariado y correlación de Spearman.

Resultados: Para los indicadores de crecimiento, $82 \%$ presentó talla e índice de masa corporal adecuado para la edad, las mujeres entre 12 y 14 años el componente predominante fue la masa adiposa, en contraste, para los hombres

'Universidad CES. Medellín, Colombia. entre 15 y 17 años fue la masa muscular, independientemente del método antropométrico utilizado. Se encontró una correlación positiva alta entre la edad y la masa magra, masa muscular, y contenido mineral óseo para los hombres. La mesomorfia predominó en hombres independientemente de la edad.

Conclusiones: El fraccionamiento de la masa corporal por cinco componentes y DEXA son métodos más específicos, evalúan tejido muscular, óseo y adiposo que están directamente relacionados con la evaluación de estado de salud y desempeño físico de cualquier individuo, además con implicaciones importantes en la calidad de vida debido a su impacto metabólico y fuerza funcional.

Palabras clave: cineantropometría, Colombia, escolares, índice de masa corporal.

Keywords: Dietary Nutritional Quality; Dietary Diversity; Micronutrients; Nutrient Adequacy, Women of Childbearing Age.

\section{Abstract ID: 193}

\section{INTERVENCIÓN NUTRICIONAL DE ADULTOS EN ESTADO CRÍTICO DIAGNOSTICADOS CON SARS-COV-2 EN LA UNIDAD DE CUIDADOS INTENSIVOS DEL HOSPITAL UNIVERSITARIO NACIONAL DE COLOMBIA. REPORTE DE CASOS}

\author{
NUTRITIONAL INTERVENTION OF CRITICALLY ILL ADULTS DIAGNOSED WITH SARS-COV-2 IN THE INTENSIVE CARE UNIT OF THE NATIONAL \\ UNIVERSITY HOSPITAL OF COLOMBIA. CASE REPORT
}

Doris Amanda Ramírez de Peña', Luisa Fernanda Cantillo Barrera'.

Introducción: Finalizando el año 2019 aparece un nuevo coronavirus conocido como SARS-CoV-2 (COVID-19) Síndrome Respiratorio Agudo Severo originario de Wuhan (China). Se propaga por medio de gotas respiratorias, emanadas al hablar, de estornudos y tos. Afecta primordialmente a adultos mayores con enfermedades crónicas. Por ser una enfermedad nueva es de gran importancia conocer el tratamiento nutricional y su evolución en una Unidad de Cuidado intensivo.

Objetivo: Describir la Terapia Médico Nutricional de pacientes diagnosticados con SARS-CoV-2 en la Unidad de Cuidados Intensivos UCl del Hospital Universitario Nacional (HUN).

Métodos: Se analizaron 9 historias clínicas de pacientes mayores de 60 años diagnosticados con COVID-19 con comorbilidades crónicas teniendo en

'Departamento de Nutrición y Dietética, Facultad de Medicina, Universidad Nacional de Colombia. Bogotá, Colombia. cuenta las variables edad, género, días de estancia hospitalaria, complicaciones, análisis bioquímicos, valoración y tratamientos médico y nutricional.

Resultados: Promedio edad: 63 años, 6 hombres 3 mujeres, estancia UCI:18 días, Diagnóstico nutricional: 8 pacientes con sobrepeso y obesidad 6 de ellos con diabetes e HTA y uno desnutrido, permanecieron con glucometrías, PCR y Dímero $D$ elevados, el tratamiento nutricional por sonda nasogástrica, para 4 pacientes con fórmula polimérica hiperproteica hipercalórica, 3 pacientes con polimérica normocalórica, 2 pacientes con polimérica normoproteica con fibra. Hubo 3 decesos.

Conclusiones: El conocimiento de la presentación y evolución de esta nueva patología permite proponer un tratamiento nutricional acorde con la evolución y complicaciones sistémicas presentadas.

Palabras clave: COVID-19, paciente crítico, nutrición.

Keywords: COVID-19; Critical Patient; Nutrition. 


\title{
Trabajos de investigación
}

Abstract ID: 153

\section{CAMBIOS EN LA MICROBIOTA INTESTINAL DE PACIENTES CRÍTICOS CON SEPSIS UNA SEMANA DESPUÉS DEL INGRESO A LA UNIDAD DE CUIDADOS INTENSIVOS}

\author{
CHANGES IN THE INTESTINAL MICROBIOTA OF CRITICALLY ILL PATIENTS WITH SEPSIS ONE WEEK AFTER ADMISSION TO THE INTENSIVE \\ CARE UNIT \\ Ana María Jaillier Ramírez, Beatriz Elena Valdés Duque, Nubia Amparo Giraldo Giraldo, Victoria Mesa, Janeth Barbosa Barbosa, Mónica Yepes Molina, \\ Gloria María Agudelo Ochoa.
}

Introducción: los pacientes críticos presentan alteraciones en la microbiota intestinal (MI) durante la estancia en la unidad de cuidados intensivos (UCI), pocos estudios han evaluado los cambios durante la estancia en UCI.

Objetivo: describir el cambio en la MI de pacientes críticos con sepsis entre el ingreso y una semana después de la estancia en $\mathrm{UCl}$.

Métodos: estudio observacional descriptivo. Fueron incluidos pacientes críticos con sepsis con muestra de heces al ingreso y 7 días después. La MI se evaluó secuenciando el gen $16 \mathrm{~S}$ rRNA. Se analizó abundancia relativa, alfa y beta diversidad e interacción de la MI con variables de interés.

Resultados: muestra final conformada por 28 pacientes. Al ingreso y al día 7 la Ml se caracterizó por filos y géneros patógenos. Siete días después,

Hospital San Vicente Fundación Rionegro | Colegio Mayor de Antioquia | Universidad de Antioquia | Universidad de Antioquia | Clínica las Américas | Hospital San Vicente Fundación Medellín | Universidad de Antioquia, Colombia. se observó disminución significativa en abundancia y riqueza $(p<0.05)$ y dispersión significativa de la comunidad microbiana que explicó en un 15,9\% su variabilidad $(p<0.05)$. El modelo lineal mixto mostró asociación significativa estricta de géneros a la mayoría de las variables, y asociados a los dos momentos, disminución significativa al día 7 de Peptococcus y Desulfovibrio, géneros patógenos $(p<0.05)$.

Conclusión: siete días después del ingreso a la $\mathrm{UCl}$, la MI de los pacientes críticos con sepsis disminuyó en abundancia y diversidad, y aunque se caracterizó por presencia de patógenos, una semana después mostró disminución de algunos de estos géneros. Son necesario estudios que evalúen cambios de la Ml en el mediano y largo plazo.

Palabras clave: microbiota intestinal, disbiosis, paciente crítico, secuenciación gen 16s RNA, sepsis, unidad de cuidado intensivo.

Keywords: Gut Microbiota; Dysbiosis; Critical Patient; 16s RNA Gene Sequencing; Sepsis; Intensive Care Unit.

\section{Abstract ID: 154}

\section{PROGRAMAS DE EDUCACIÓN NUTRICIONAL DIRIGIDOS A MADRES AFRICANAS DE NIÑOS EN EDAD INFANTIL: UNA REVISIÓN SISTEMÁTICA}

\author{
NUTRITION EDUCATION PROGRAMS AIMED AT AFRICAN MOTHERS OF INFANT CHILDREN: A SYSTEMATIC REVIEW
}

Byron Casanova', Victoria Arija², Cristina Jardí1,2.

Introducción: La desnutrición infantil es un importante problema epidemiológico en los países en vías de desarrollo. La educación en nutrición de las madres puede paliar esta desnutrición en sus hijos pequeños. El objetivo fue realizar una revisión sistemática para valorar el efecto de los programas de intervención en educación nutricional, dirigidos a madres africanas, sobre el estado nutricional en sus hijos de edad infantil.

Métodos: Se ha realizado una búsqueda bibliográfica en la base de datos PubMed delimitada a ensayos clínicos entre los años 2015 y 2020. Los estudios debían evaluar el impacto sobre indicadores nutricionales del niño (consumo alimentario, antropometría y/o conocimientos en nutrición).

Resultados: 15 artículos fueron seleccionados. 53\% de los estudios evaluaron el consumo alimentario de los niños, $82 \%$ las medidas antropométricas,

'Medicina Preventiva y Salud Pública, Universitat Rovira i Virgili, 43201 Reus, Spain ${ }^{2}$ Pere Virgili Institute for Health Research (IISPV), Universitat Rovira i Virgili, 43003 Tarragona, Spain
$30 \%$ los conocimientos nutricionales; en general se atribuye a los programas de educación nutricional significativas mejorías en el consumo de alimentos y nutrientes, conocimientos y prácticas dietarías en alimentación complementaria, y reducciones en las cifras de desnutrición crónica.

Conclusiones: no existe uniformidad en la evaluación de los resultados. Sin embargo, el presente estudio demostró, que solamente la educación no es suficiente para generar cambios nutricionales y que estos deberían acompañados de programas de agricultura doméstica o de suplementación nutricional para hacer efectivos los cambios y lograr una adecuada adherencia a las recomendaciones y guías de alimentación saludable.

Palabras clave: malnutrición, retraso en crecimiento, desnutrición aguda, bajo peso, programas en nutrición, revisión sistemática.

Keywords: Malnutrition; Stunting; Wasting; Underweight; Nutrition Programs; Systematic Review. 
Abstract ID: 164

\title{
INFLAMMATION MARKERS AND EXERCISE IN ADOLESCENTS WITH EXCESS ADIPOSITY: A SECONDARY ANALYSIS OF A RANDOMIZED CLINICAL TRIAL "HEPAFIT STUDY"
}

\author{
MARCADORES INFLAMATORIOS Y EJERCICIO EN ADOLESCENTES CON EXCESO DE ADIPOSIDAD: UN ANÁLISIS SECUNDARIO DEL \\ ENSAYO CLÍNICO CONTROLADO "ESTUDIO HEPAFIT"
}

Robinson Ramírez-Vélez, Mikel Izquierdo, Antonio García-Hermoso, Sara Palomino-Echeverría, Katherine González-Ruíz, Enrique Santamaría, Joaquín Fernández-Irigoyen.

Objective: Obesity-related metabolic risk factors in adolescents with excess body adipose may be associated with systemic low-grade inflammation, and therefore we investigated whether a 6-month exercise training altered markers of inflammation.

Methods: Secondary analyses of a randomized controlled exercise-based intervention trial (September 2017 to December 2018). Adolescents aged 11 to 17 years with excess weight (overweight and obesity) status and/or excess of adipo-

Navarrabiomed, Complejo Hospitalario de Navarra (CHN)-Universidad Pública de Navarra (UPNA), IdiSNA, Pamplona (Spain). | Navarrabiomed, Complejo Hospitalario de Navarra (CHN)-Universidad Pública de Navarra (UPNA), IdiSNA, Pamplona (Spain). | Navarrabiomed, Complejo Hospitalario de Navarra (CHN)-Universidad Pública de Navarra (UPNA), IdiSNA, Pamplona (Spain). | Translational Bioinformatics Unit (TransBio), Navarrabiomed, Navarra Health Department, Public University of Navarra, Navarra Institute for Health Research (IdiSNA), Pamplona, 31008, Spain. | Grupo de Ejercicio Físico y Deportes, Vicerrectoría de Investigaciones, Facultad de Salud, Universidad Manuela Beltrán, Bogotá 110231, Colombia | Proteored- Institute of Health Carlos III (ISCIII), Clinical Neuroproteomics Unit, Navarrabiomed, Navarra Health Department, Public University of Navarra, Navarra Institute for Health Research (IdiSNA), Pamplona, 31008, Spain | Proteored-Institute of Health Carlos III (ISCIII), Clinical Neuroproteomics Unit, Navarrabiomed, Navarra Health Department, Public University of Navarra, Navarra Institute for Health Research (IdiSNA), Pamplona, 31008, Spain. sity (body fat $>30 \%$ ). The adolescents were randomly assigned to the following 4 groups for 6 months: (1) standard physical education lessons, as a control (CTRL); (2) high-intensity physical education class (HIPE); (3) low-to-moderate intensity physical education class (LIPE); (4) a combined group (PLUS group). Inflammatory markers and immune molecules including chemokines, cytokines, and growth factors were determined by cytokine antibody array.

Results: Of the 120 randomly assigned participants, 95 were included in the analysis. Serum inflammatory levels such as MIP-1b (CCL4), RANTES (CCL5), BCL (CXC13), Eotaxin (CCL11), PARC (CCL19), and FGF-6 (Fibroblast Growth Factor-6) decreased significantly in the HIPE and PLUS groups $(p<0.05)$, supporting the antiinflammatory effect of these two training programs.

Conclusions: Implementing a 6-month physical exercise program in overweight/obese adolescents, based on HIPE and PLUS groups, significantly change several circulating inflammatory levels. Interventions involving supervised physical exercise may reduce the associated effects of systemic low-grade inflammation, thus preventing the development of obesity-related metabolic diseases in adolescents with overweight/obesity. ClinicalTrials.gov registration (NCT02753231).

Keywords: Exercise; Inflammation; Youth; Transient Elastography.

Palabras clave: ejercicio, inflamación, adolescencia, elastografía transitoria.

Abstract ID: 165

\section{LIPIDOMIC PROFILE FROM PHYSICALLY FRAIL AND ROBUST OLDER ADULTS AT HOSPITAL ADMISSION}

\author{
PERFIL LIPIDÓMICO DE ADULTOS MAYORES FÍSICAMENTE FRÁGILES Y ROBUSTOS AL MOMENTO DE LA ADMISIÓN HOSPITALARIA
}

Robinson Ramírez-Vélez, Mikel Izquierdo, Nicolás Martínez-Velilla, Mikel L. Sáez de Asteasu, Antonio García-Hermoso, Fabricio Zambom-Ferraresi.

Background: Identifying serum biomarkers that can predict physical frailty in older adults would have tremendous clinical value for primary care, as this condition is inherently related to poor quality of life and premature mortality. We compared the serum lipid profile of physically frail and robuster older adults to identify specific lipid biomarkers that could be used to assess physical frailty in older patients at hospital admission.

Methods: Seventy older adults (54.3\% women), mean (SD) age 86.8 (4.8) years, were classified as physically frail $(n=37)$ or robust $(n=33)$ based on scores from the Short Physical Performance Battery. Plasma lipidomic profiles were

Navarrabiomed, Complejo Hospitalario de Navarra (CHN)-Universidad Pública de Navarra (UPNA), IdiSNA, Pamplona (Spain). | Navarrabiomed, Complejo Hospitalario de Navarra (CHN)-Universidad Pública de Navarra (UPNA), IdiSNA, Pamplona (Spain). | Department of Geriatric Medicine, Complejo Hospitalario de Navarra, Irunlarre, Pamplona, Spain. Navarrabiomed, Complejo Hospitalario de Navarra (CHN)-Universidad Pública de Navarra (UPNA), IdiSNA, Pamplona (Spain). | Navarrabiomed, Complejo Hospitalario de Navarra (CHN)- Universidad Pública de Navarra (UPNA), IdiSNA, Pamplona (Spain).| Navarrabiomed, Complejo Hospitalario de Navarra (CHN)-Universidad Pública de Navarra (UPNA), IdiSNA, Pamplona (Spain). examined using ultra-high performance liquid chromatography coupled to mass spectrometry. Univariate analysis was used to identify the lipid metabolites differing significantly between physically frail and robust older adults.

Results: We identified a panel of 11 metabolites whose levels were significantly lower in physically frail adults than in robuster older peers: triglycerides $T G(40: 0), T G(42: 0), T G(44: 0), T G(45: 0)$ and $T G(46: 0)$; cholesteryl esters $C E(18: 3)$ and $C E(20: 5)$; phosphatidylcholines $P C(16: 0 / 20: 5), P C(18: 3 / 18: 3)$ and $P C(38: 5)$; and phosphatidylethanolamine $P E(20: 4 / 18: 2)$ Receiver-operating curve characteristic analysis revealed that $P C$ (16:0/20:5) had an acceptable degree of diagnostic accuracy, with an area under the curve of 0.70 .

Conclusions: Targeted lipidomics supports the existence of a lipidomic profile in physically frail hospitalized older patients. Phosphatidylcholine $P C(16: 0 / 20: 5)$ had an acceptable level of accuracy to discriminate physical frailty in older people and, therefore, might be a useful biomarker in clinical practice.

Keywords: Frailty; Lipidomic; Phosphatidylcholines; Biomarker; Older Adults.

Palabras clave: fragilidad, lipidómico, fosfatidilcolinas, biomarcador, adultos mayores. 
Abstract ID: 174

\title{
RELACIÓN ENTRE LA MICROBIOTA INTESTINAL Y LOS ÁCIDOS GRASOS DE CADENA CORTA EN PACIENTES CRÍTICOS CON SEPSIS DURANTE SU ESTANCIA EN LA UNIDAD DE CUIDADOS INTENSIVOS
}

\author{
RELATIONSHIP BETWEEN GUT MICROBIOTA AND SHORT CHAIN FATTY ACIDS IN CRITICAL PATIENTS WITH SEPSIS DURING THEIR STAY IN \\ THE INTENSIVE CARE UNIT
}

Luz Dary Vásquez Guarín'.

Introducción: el paciente crítico con sepsis presenta alteraciones en la Microbiota Intestinal (MI) que pueden afectar la producción de Ácidos Grasos de Cadena Corta (AGCC) y condicionar la respuesta inmune e inflamatoria.

Objetivo: establecer la relación entre la MI y la concentración de AGCC en pacientes críticos con sepsis.

Métodos: estudio observacional descriptivo. Incluyó 15 pacientes críticos con sepsis que tuviesen una muestra de heces para MI y otra para AGCC del mismo día o con un día de diferencia. La MI se evaluó por secuenciación de las regiones V3-V4 del gen 16S rRNA y se reportó la taxonomía a nivel de filo y género. Los AGCC fueron cuantificados por cromatografía de gases y para establecer la relación entre la MI y los AGCC se realizó la prueba de correlación de Kendall.

Resultados: se identificaron a nivel de filo principalmente Bacteroidetes, Firmicutes y Proteobacterias; los géneros bacterianos más representativos

'Universidad de Antioquia, Colombia fueron Bacteroides, Pseudomonas, Escherichia-Shigella, Prevotella, Klebsiella, Mycoplasma y Parabacteroides. Se evidenciaron niveles bajos de AGCC y una correlación inversa entre la edad y el ácido isobutírico $(p=0,01)$. Todos los AGCC se correlacionaron positivamente con un género de la familia Ruminococcaceae y de forma negativa con el género Enterococcus.

Conclusiones: en pacientes críticos con sepsis la composición de la MI se altera con predominio de microorganismos patógenos y los principales AGCC se encuentran en bajas concentraciones; a mayor riqueza de microorganismos comensales, mayor es la concentración de AGCC, mientras que, a mayor presencia de patógenos la concentración es menor.

Palabras clave: microbiota intestinal, paciente crítico, sepsis, unidad de cuidados intensivos, ácidos grasos de cadena corta.

Keywords: Gut Microbiota; Critical IIIness; Sepsis; Intensive Care Unit; Short Chain Fatty Acids.

Abstract ID: 179

\section{DETERMINACIÓN DE LA PREVALENCIA DE RIESGO NUTRICIONAL EN PACIENTES ADULTOS QUE INGRESAN A HOSPITALIZACIÓN EN LA CUC DURANTE LOS AÑOS DE 2016 A 2018}

\author{
DETERMINATION OF THE PREVALENCE OF NUTRITIONAL RISK IN ADULT PATIENTS ADMITTED TO HOSPITALIZATION IN THE CUC DURING
} THE YEARS 2016 TO 2018

Diana Julieth Vargas Moreno'.

Introducción: La desnutrición es catalogada como un problema de salud pública, la identificación del riesgo nutricional de manera oportuna y el tratamiento de la desnutrición al ingreso o durante la hospitalización lleva a mejores resultados hospitalarios y mayor calidad de vida de los pacientes.

Objetivo general: Determinar la prevalencia de riesgo nutricional en pacientes adultos que ingresaron a hospitalización en la CUC durante los años 2016 a 2018.

Métodos: Se incluyeron pacientes adultos que ingresaron a hospitalización entre los años 2016 a 2018 en una clínica de 4 nivel, se excluyeron pacientes con alteración del estado de conciencia o trastornos cognitivos, gestantes, y pacientes con limitación del esfuerzo terapéutico o en fin de vida. Se utilizó la escala

'Grupo de Investigación en Nutrición Clínica, Fundación Universitaria. Sanitas, Clínicas Colsanitas, Colombia.
MST, se realizó estadística descriptiva y un análisis de supervivencia tomando la estancia hospitalaria como desenlace por el método de Kaplan-Meier.

Resultados: Se analizaron 26122 registros, con riesgo nutricional moderado a severo el $12.4 \%$ ( $n=3249)$ y riesgo leve el $20,3 \%(n=5284)$, de los pacientes con riesgo moderado a severo se encontró algún grado de desnutrición en el $93 \%$. Los pacientes con riesgo nutricional moderado a severo tuvieron una mediana de estancia de 4 días más (RIQ 4-12 días) al compararlo con los pacientes sin riesgo nutricional. El riesgo nutricional moderado a severo, el género masculino, la hospitalización inicial en $\mathrm{UCl}$ y la desnutrición fueron las categorías que se asociaron en una estancia hospitalaria prolongada.

Conclusiones: La identificación del riesgo nutricional en el ámbito hospitalario nos permite realizar intervenciones oportunas impactando en desenlaces hospitalarios como la estancia hospitalaria.

Palabras clave: tamización, desnutrición, evaluación nutricional.

Keywords: Screening; Malnutrition; Nutritional Assessment.

Abstract ID: 183

\section{EVALUACIÓN DE LAS CARACTERÍSTICAS OPERATIVAS DE LAS HERRAMIENTAS DE TAMIZAJE NUTRICIONAL NUTRISCORE Y MALNUTRITIONAL SCREENING TOOL COMPARADAS CON LA VALORACIÓN NUTRICIONAL COMPLETA, EN EL PACIENTE ONCOLÓGICO ADULTO HOSPITALIZADO EN LA CLÍNICA UNIVERSITARIA COLOMBIA}

\author{
EVALUATION OF THE OPERATIONAL CHARACTERISTICS OF THE NUTRITIONAL SCREENING TOOLS NUTRISCORE AND MALNUTRITIONAL \\ SCREENING TOOL COMPARED WITH THE COMPLETE NUTRITIONAL ASSESSMENT, IN ADULT CANCER PATIENTS HOSPITALIZED AT CLÍNICA \\ UNIVERSITARIA COLOMBIA
}

Daniela Alejandra Acero Alfonso 1,2, Ricardo Alfonso Merchán Chaverra ${ }^{3}$, Yeny Marjorie Cuellar Fernández ${ }^{3}$, Jorge Alexis Medina Parra ${ }^{3}$.

Introducción: La detección temprana del riesgo nutricional en el paciente oncológico cobra relevancia, dado que permite una intervención nutricional oportuna a fin de mitigar el progreso hacia la caquexia tumoral y evitar complicaciones que generen mayor deterioro en el estado de salud del individuo.

Objetivo general: Evaluar las características operativas de las herramientas de tamizaje nutricional Nutriscore y Malnutritional Screening Tool comparadas con la valoración nutricional completa aplicada en pacientes oncológicos adultos hospitalizados en la Clínica Universitaria Colombia.

Métodos: Se incluyeron pacientes adultos oncológicos, que ingresaron al servicio de hospitalización de Clínica Universitaria Colombia y se encontraban en las primeras 24 horas de internación., fueron evaluados utilizando Nutriscore, MST y Valoración Nutricional Completa. Para la determinación de 
las características de las pruebas diagnósticas, se calculó sensibilidad, especificidad, LR+ y LR-, para las herramientas de tamizaje, empleando la Valoración Nutricional Completa como método de referencia.

Resultados: Se evaluaron 439 pacientes. Utilizando como método de referencia la Valoración Nutricional Completa, Nutriscore obtuvo una sensibilidad de $44.6 \%$ y una especificidad de $97.1 \%$, mientras que MST supero estos valo-

'Clínica Universitaria Colombia. Bogotá, Colombia.

${ }^{2}$ Clínica Colsanitas, Bogotá, Colombia.

${ }^{3}$ Fundación Universitaria Sanitas (UNISANITAS). Bogotá, Colombia. res con una sensibilidad de $74.9 \%$ y una especificidad de $94.3 \%$. El mejor desempeño de MST en comparación con Nutriscore fue confirmado por el análisis de la curva de características operativas, con valores de área bajo la curva de 0.84 (IC 95\% 0.81-0.88) para MST y 0.71 (IC 95\% 0.67-0.74) para Nutriscore.

Conclusión: Se propone MST como herramienta de tamizaje nutricional de los pacientes oncológicos hospitalizados dado que presenta mejores características operativas, comparado con Nutriscore.

Palabras clave: tamizaje, desnutrición, neoplasia.

Keywords: Screening; Malnutrition; Neoplasm.

Abstract ID:185

VARIACIÓN DE PARÁMETROS ULTRASONOGRÁFICOS EN PACIENTES CRÍTICAMENTE ENFERMOS CON SARS-COV-2 / COVID-19

\author{
VARIATION OF ULTRASONOGRAPHIC PARAMETERS IN CRITICALLY ILL PATIENTS WITH SARS-COV-2 / COVID-19
}

Johan Sebastián Torres Mora', Ricardo Alfonso Merchán Chaverra², Yeny Marjorie Cuellar Fernández², Jorge Alexis Medina Parra

Introducción: El 5\% de los pacientes diagnosticados con SARS-CoV-2 (COVID-19) requieren ventilación mecánica invasiva los cuales presentan pérdida muscular, unos en mayor grado que otros, dependiendo de diferentes variables según su condición. Como objetivo fue identificar la pérdida de masa muscular del recto femoral y vasto interno en los pacientes diagnosticados con COVID-19 que requirieron ventilación mecánica invasiva.

Método: Esto fue un estudio longitudinal retrospectivo de bajo riesgo, en la Clínica Infantil Santa María del Lago, en Bogotá, en el periodo de junio a agosto de 2020. Todos los pacientes evaluados fueron mayores de 18 años con diagnóstico de SARS-CoV-2.

${ }^{1}$ Clínica Infantil Santa María del Lago - Clínica Colsanitas. Bogotá, Colombia. ${ }^{2}$ Fundación Universitaria Sanitas (UNISANITAS). Bogotá, Colombia.
Resultados: Se obtuvo una muestra de 39 pacientes con un promedio de 13 días en la UCl, la mayoría de proporción de los pacientes presentaban exceso de peso. Se identificó un cubrimiento del $80.4 \%$ de las metas calóricas y un $82 \%$ de las metas proteicas. Se identificó pérdida del recto femoral en la primera semana del $25.9 \%$, en la segunda semana una pérdida del $37.4 \%$ y en la tercera semana $56.3 \%$ y del vasto interno con una pérdida del $24.8 \%$, en la segunda semana del $26.8 \%$ y en la tercera semana del $49.9 \%$.

Conclusión: se identificó una pérdida muscular en los pacientes diagnosticados por SARS-CoV-2 con un promedio diario aproximadamente del $3 \%$.

Palabras clave: SARS-CoV-2, malnutrición, ultrasonografía.

Keywords: SARS-CoV-2; Malnutrition; Ultrasonography.

Abstract ID: 190

\title{
DOBLE CARGA NUTRICIONAL EN HOGARES COLOMBIANOS: UNA APROXIMACIÓN DESDE LOS DETERMINANTES SOCIALES EN 2010
}

DOUBLE BURDEN OF MALNUTRITION IN COLOMBIAN HOUSEHOLDS: AN APPROACH FROM SOCIAL DETERMINANTS IN 2010

Yenny Paola Rueda Guevara', Catalina González Uribe’.

Introducción: En la actualidad se observa la coexistencia de malnutrición por déficit y por exceso en los hogares. De acuerdo con la Encuesta Nacional de Situación Nutricional ENSIN, el retraso en talla en menores de cinco años ha disminuido del $16 \%$ en 2005 a 10.8\% en 2015; mientras que el exceso de peso en adultos, para el mismo periodo, aumentó de $45,9 \%$ a $56,4 \%$.

Objetivo: Establecer cuáles son los determinantes sociales de la coexistencia de retraso en talla en menores de cinco años y exceso de peso materno en hogares colombianos para el 2010.

Métodos: Estudio de tipo descriptivo de corte transversal a partir de fuentes secundarias: ENSIN 2010. La muestra incluyó 10.423 parejas madre-hijo. Se

'Fundación Santa Fe de Bogotá, Universidad de Los Andes. Bogotá, Colombia. estimó la prevalencia de DCN en hogares y se estimó un modelo de regresión logística con análisis de sensibilidad.

Resultados: La prevalencia de DCN en los hogares se estableció en $4.66 \%$, con mayor prevalencia a nivel urbano y en hogares con niveles educativos bajos de la madre. Se encontró asociación entre la DCN y el bajo nivel educativo de la madre, minorías étnicas, sexo de la hija mujer y número de orden de nacimiento del hijo.

Conclusiones: Existen determinantes sociales estructurales e intermedios que contribuyen con el desarrollo de la doble carga nutricional a nivel de hogar, lo que sugiere la creación estrategias que impacten la desnutrición infantil y el sobrepeso materno.

Palabras clave: desnutrición, sobrepeso, determinantes sociales de la salud.

Keywords: Malnutrition; Overweight; Social Determinants of Health. 
Abstract ID: 199

\title{
CARACTERÍSTICAS DEL CRECIMIENTO EN EL PACIENTE PEDIÁTRICO CON FENILCETONURIA, UNA REVISIÓN DE LITERATURA
}

\author{
GROWTH CHARACTERISTICS IN PEDIATRIC PATIENTS WITH PHENYLKETONURIA, A LITERATURE REVIEW
}

Luisa Fernanda Torres Laiton?.

Introducción: La fenilcetonuria es un error innato del metabolismo de la fenilalanina (Phe) causado por la deficiencia de la enzima fenilalanina hidroxilasa, esta patología se caracteriza por la elevación en las concentraciones de Phe generando daño neurológico. La intervención nutricional busca controlar los rangos de Phe, favorecer el crecimiento y desarrollo y prevenir los efectos secundarios de la enfermedad. El régimen nutricional se basa en una dieta restrictiva que puede tener efectos potencialmente negativos en el crecimiento y desarrollo.

Objetivos: Describir las características del crecimiento en los pacientes pediátricos con $\mathrm{PKU}$ intervenidos con un régimen nutricional.

'Universidad Nacional de Colombia. Bogotá, Colombia.
Método: Se realizó una búsqueda de literatura de artículos con base a las directrices de la declaración PRISMA. Se incluyeron estudios retrospectivos longitudinales y estudios transversales, con una muestra mayor a 50 pacientes seguidos por más de 1 año.

Resultados: Los estudios valoraron el crecimiento en z-scores de peso, talla e IMC, se reporta una tendencia a la disminución de la talla en el grupo con PKU, respecto al peso los resultados varían entre la disminución y la normalidad, con una tendencia al sobrepeso en las niñas. Los pacientes con hiperfenilalaninemia tuvieron valores normales.

Conclusiones: Los resultados de los estudios son heterogéneos y demuestran las características variables del crecimiento en niños con PKU.

Palabras clave: fenilcetonuria, crecimiento, errores innatos del metabolismo.

Keywords: Phenylketonuria; Growth; Inborn Errors of Metabolism.

\section{Protocolos}

Abstract ID: 152

\section{CALIDAD MICROBIOLÓGICA DE LOS ALIMENTOS OFERTADOS EN LA UNIVERSIDAD EL BOSQUE Y SUS ALREDEDORES: PROTOCOLO}

\author{
MICROBIOLOGICAL QUALITY OF FOOD OFFERED AT EL BOSQUE UNIVERSITY AND SURROUNDING AREAS: PROTOCOL
}

María Angélica González Cruz', Luis Alejandro García Rairán'.

\begin{abstract}
Introducción: Restaurantes, tiendas y puestos de comida representan un entorno importante para el consumo de alimentos mientras se desempeñan funciones académicas o laborales. Siendo frecuente que se produzcan enfermedades transmitidas por alimentos (ETAs) ocasionadas por falta de buenas prácticas de manufactura (BPM), por la ingestión de alimentos y/o bebidas contaminados con microorganismos patógenos que afectan la salud del consumidor, individual o colectivamente. En Colombia, las ETAs afectan principalmente al grupo etario entre 20-49 años seguido de población de 10-19 años. La mayoría de estudios sobre ETAs en comunidades universitarias, no consideran las propias instalaciones de la universidad o lo hacen parcialmente, son aplicables sólo a puestos ambulantes adyacentes a las universidades. De esta manera la siguiente investigación busca detectar y analizar los microorganismos que se encuentran en los alimentos de mayor consumo por la comunidad universitaria, su cantidad para definir si son patógenos y brindar
\end{abstract}

'Universidad El Bosque. Bogotá, Colombia. sugerencias con respecto a las BPM, además, permitirá ajustar estrategias institucionales para la prevención de las ETAs en estudiantes de la Universidad El Bosque (UEB).

Objetivos: describir la calidad microbiológica de los alimentos ofertados en la UEB y sus alrededores durante Agosto del 2021. Aislando y caracterizando los microorganismos Gram- y Gram+ por pruebas bioquímicas en los productos cárnicos, panadería, lácteos y sus derivados, bebidas no alcohólicas, gaseosas o carbonatadas, frutas y hortalizas ofertados en la UEB y en un perímetro de 200 metros.

Resultados esperados: se espera aislar con éxito microorganismos enteropatógenos. Un Trabajo de grado que conducirá a la obtención del título de médico cirujano.

Palabras claves: UFC, alimentos, microorganismo, buenas prácticas de manufactura, ETA.

Keywords: Colony Forming Units; Food; Microorganism; Good Manufacturing Practices; Foodborne Diseases. 
Abstract ID: 157

\title{
NIVELES DE BISFENOL A URINARIO Y SU RELACIÓN CON LOS INDICADORES ANTROPOMÉTRICOS EN PACIENTES HOSPITALIZADOS DEL INSTITUTO NACIONAL DE SALUD DEL NIÑO
}

\author{
URINARY BISPHENOL A LEVELS AND THEIR RELATIONSHIP WITH ANTHROPOMETRIC INDICATORS IN HOSPITALISED PATIENTS AT THE \\ NATIONAL INSTITUTE OF CHILD HEALTH
}

Marco Antonio Sánchez Gaspar' ${ }^{1}$, Heleen Alicia Chaupin Cuellar' , Yadira Lilia Cairo Arellano².

\begin{abstract}
Introducción: El Bisfenol A (BPA), conocido como disruptor endocrino, se encuentra en el plástico, derivados y fuentes de contacto. Encontrándose también en el medio ambiente (agua, aire, suelo) y fuentes de contacto de uso diario por el hombre (alimentos, equipos médicos) dentro las principales vías de contaminación.

Objetivo: Determinar la relación entre los niveles de Bisfenol A urinario con los Indicadores Antropométricos en pacientes del Instituto Nacional de Salud del Niño.

Métodos: Estudio de tipo Cuantitativo, Analítico, Observacional, Transversal y Prospectivo con muestreo probabilístico. Lugar: Instituto de Salud del Niño, San Borja - Perú.

Muestra: Formada por 50 pacientes pediátricos con enfermedad renal crónica. Para el análisis estadístico se utilizará el programa STATA 16.0. Para el
\end{abstract}

'Instituto Nacional de Salud del Niño. San Borja, Perú. ${ }^{2}$ Instituto Nacional de Salud del Niño. San Borja, Perú. análisis bivariado se hará uso de a prueba T Student en variables cuantitativas, Chi Cuadrado para variables cualitativas. Análisis multivariado: regresión logística binomial. La determinación del Bisfenol A urinario total y conjugado se realizará por medio de la espectrometría de masa GS-MS; así como el uso de la antropometría para la medición de los indicadores antropométricos.

Perspectivas y Resultados esperados: Será un estudio pionero en el campo de la toxicología, pediatría y nutrición, que busque explicar la posible relación entre los niveles de BPA y los indicadores antropométricos en pacientes pediátricos en el Perú. Se espera encontrar una relación estadísticamente significativa positiva. Los resultados servirán como base para el desarrollo de investigaciones de mayor calidad metodológica y rigurosidad científica como guías de práctica clínica.

Palabras clave: bisfenol A, antropometría, pediatría, estado nutricional. Keywords: Bisphenol A; Anthropometry; Paediatrics; Nutritional Status.

Abstract ID: 166

\section{ESTUDIO MULTICÉNTRICO SOBRE EL IMPACTO DEL SOPORTE NUTRICIONAL EN EL ESTADO NUTRICIONAL EN PACIENTES CRÍTICOS CON COVID-19 EN HOSPITALES DEL PERÚ}

\author{
MULTICENTRE STUDY ON THE IMPACT OF NUTRITIONAL SUPPORT ON THE NUTRITIONAL STATUS OF CRITICALLY ILL PATIENTS WITH \\ COVID-19 IN PERUVIAN HOSPITALS \\ Marco Antonio Sánchez Gaspar', Heleen Alicia Chaupin Cuellar', Luis Roberto Castillo Arellano', Julio Rafael Kiwaki Gómez’ .
}

Introducción: Actualmente el Perú es uno de los países en América Latina con mayores estragos y víctima de la pandemia a nivel mundial por el virus del SARS-CoV-2. Lo cual refleja el aumento paulatino del número de infectados y de defunciones en todo el país por el COVID-19.

Objetivo: Determinar el impacto del Soporte Nutricional en el Estado Nutricional en pacientes críticos con COVID-19 en Hospitales del Perú.

Métodos: Estudio retrospectivo con muestreo probabilístico. La población estará formada por pacientes críticos con COVID-19 en hospitales del Perú a nivel nacional. Para el análisis estadístico se utilizará el programa STATA 16.0; para el caso del análisis univariado: promedios para variables continuas, frecuencias para variables categóricas. Análisis bivariado: T Student en variables cuantitativas, Chi Cuadrado para variables cualitativas. Análisis multivariado: regresión logística binomial.

${ }^{1}$ Hospital Daniel Alcides Carrión. Callao, Perú.
Perspectiva y resultados esperados: El presente estudió contribuirá abordar la problemática nacional en el campo de la salud, ocasionada por la pandemia del COVID-19, en los centros hospitalarios del Perú. Así mismo busca demostrar la importancia de la adecuada praxis del soporte nutricional. Es por ello que será un estudio pionero en el campo de la nutrición clínica en el Perú y América Latina, enfatizando el papel de la terapia nutricional a cargo del equipo multidisciplinario de salud en el paciente crítico. Se espera un impacto positivo y significativo entre los indicadores del soporte nutricional y el estado nutricional.

Palabras clave: COVID-19, SARS-CoV-2, terapia nutricional, estado nutricional, cuidado crítico.

Keyword: COVID-19; SARS-CoV-2; Nutritional Therapy; Nutritional Status, Critical Care.

Abstract ID: 168

\section{DETERMINACIÓN DEL VALOR PRONÓSTICO DEL ÁNGULO DE FASE TOMADO POR BIOIMPEDANCIA SOBRE LA SUPERVIVENCIA Y MALNUTRICIÓN EN PACIENTES DIAGNOSTICADOS CON LA COVID-19 EN LA CLÍNICA CARTAGENA DEL MAR DE LA CIUDAD DE CARTAGENA DE INDIAS, COLOMBIA, 2021}

\author{
DETERMINATION OF THE PROGNOSTIC VALUE OF THE PHASE ANGLE MEASURED BY BIOIMPEDANCE ON SURVIVAL AND MALNUTRITION \\ IN PATIENTS DIAGNOSED WITH COVID-19 AT THE CARTAGENA DEL MAR CLINIC IN CARTAGENA DE INDIAS, COLOMBIA, 2021
}

M. Díaz-Rincón, F. Hernández-López, OL Lora-Díaz, VJ Abauu-Rueda, G. Uribe-Gil, JP Morales-Basto.

Introducción: el ángulo de fase (AF) es un parámetro de bioimpedancia (BIA) que ha demostrado tener un adecuado valor predictivo sobre el diagnóstico y el pronóstico clínico.
Objetivo: determinar el valor pronóstico del AF tomado por BIA sobre la supervivencia y malnutrición en pacientes hospitalizados con diagnóstico de COVID-19 de la Clínica CM de la Ciudad de Cartagena. 
Métodos: estudio observacional analítico de cohorte, cuya recolección de datos se realizará entre junio y julio del 2021 a través de un cuestionario de caracterización. Las mediciones antropométricas y de la composición corporal a través de BIA serán llevadas a cabo por personal capacitado. Se construirán dos modelos multivariables; uno de riesgo proporcional de Cox y otro modelo de regresión logística para establecer la asociación entre el AF y el estado nutricional.
Perspectivas y resultados esperados: este estudio pretende contribuir en el aporte de evidencia científica frente al valor pronóstico del AF en el pronóstico clínico y estado nutricional en pacientes con el virus del SARS-CoV-2.

Palabras clave: desnutrición, impedancia eléctrica, análisis de supervivencia, COVID-19.

Keywords: Malnutrition; Electric Impedance; Survival Analysis; COVID-19.

Abstract ID:175

\title{
DESCRIPCIÓN DE SARCOPENIA EN PACIENTES HOSPITALIZADOS E INTERVENIDOS POR EL GRUPO DE SOPORTE METABÓLICO Y NUTRICIONAL DE LA FUNDACIÓN SANTA FE EN BOGOTÁ
}

\author{
DESCRIPTION OF SARCOPENIA IN HOSPITALIZED PATIENTS WITH AN INTERVENTION OF THE METABOLIC AND NUTRITIONAL SUPPORT \\ GROUP OF FUNDACIÓN SANTA FE IN BOGOTÁ
}

Ana Milena Sierra Gómez'

Introducción: En la última década se ha avanzado en la identificación y tratamiento de personas con sarcopenia, lo que ha permitido definir las características del músculo y su papel en la salud y enfermedad. Actualmente, la sarcopenia está asociada con una mayor mortalidad y estancia hospitalaria en personas mayores.

Objetivo general: Determinar la presencia de sarcopenia en pacientes hospitalizados e intervenidos por el grupo de soporte metabólico y nutricional de la Fundación Santa Fe en Bogotá en el periodo comprendido de Febrero a Agosto de 2021.

Objetivos específicos: Describir las características demográficas de la población estudio. Medir fuerza, masa muscular y velocidad de la marcha en la

'Fundación Santa Fe de Bogotá. Bogotá, Colombia. población estudio. Describir factores de riesgo conocidos en pacientes diagnosticados con sarcopenia. Caracterizar prescripción, composición y duración de la nutrición artificial en pacientes con sarcopenia.

Métodos: Hipótesis: Encontrar una incidencia baja o alta de sarcopenia de diversas causas. Estudio: Observacional descriptivo prospectivo. Muestra: No probabilística por conveniencia. Criterios de inclusión: Personas mayores de 18 años en manejo con soporte metabólico. Criterios de exclusión: Embarazadas o lactantes, personas amputadas, con deterioro cognitivo, con implantes electrónicos, materiales de osteosíntesis, ascitis, edema, con esteroides.

Perspectiva: Se espera mayor prevalencia de sarcopenia en adultos mayores y en mujeres.

Palabras claves: Sarcopenia, bioimpedancia, nutrición.

Keywords: Sarcopenia; Bioimpedance; Nutrition

\section{Abstract ID: 186 \\ ASOCIACIÓN ENTRE EL ESTADO NUTRICIONAL Y EL DESEMPEÑO COGNITIVO EN UN GRUPO DE ADULTOS MAYORES DEL MODELO DE ATENCIÓN INTEGRAL SERMÁS - IPS UNIVERSITARIA}

\author{
ASSOCIATION BETWEEN NUTRITIONAL STATUS AND COGNITIVE PERFORMANCE IN A GROUP OF ELDERLY ADULTS FROM THE SERMÁS - \\ IPS UNIVERSITARIA COMPREHENSIVE CARE MODEL
}

Mateo Londoño Pereira'

Introducción: La demencia es una de las principales causas de morbimortalidad mundial, generalmente causada por enfermedades neurodegenerativas. En la actualidad hay alrededor de 50 millones de casos y, con el envejecimiento poblacional, esta cifra podría triplicarse para 2050. En ausencia de una cura, se enfatiza en la prevención primaria de este síndrome. Se ha encontrado una asociación entre la malnutrición y el desarrollo de alteración cognitiva y demencia en adultos mayores (AM), especialmente en aquellos con enfermedades crónicas no transmisibles. Identificar y corregir oportunamente cualquier forma de malnutrición y mantener un adecuado estado nutricional (EN) podría preservar la función cognitiva de AM y prevenir el desarrollo de demencia

Objetivo: Analizar la asociación entre el estado nutricional y el desempeño cognitivo en una población de adultos mayores pertenecientes al modelo SerMás de la IPS Universitaria.

'Escuela de Nutrición y Dietética, Universidad de Antioquia. Medellín, Colombia.
Métodos: Estudio observacional, transversal, de tendencia analítica, en una muestra representativa (n:234) de AM del modelo SerMás- IPS Universitaria. Se hará una aleatorización simple por listas para el reclutamiento con criterios de selección, a los interesados se hará consentimiento informado, aprobado por comité de ética. Se clasificará el EN por Mini Nutritional Assessment, IMC y circunferencia de cintura como malnutrición, riesgo de malnutrición, estado nutricional normal, sobrepeso, obesidad y obesidad abdominal. El desempeño cognitivo se caracterizará por medio del test MoCA. Los análisis estadísticos se realizarán en los software $\mathrm{R}$ y Jamovi.

Resultados esperados: Se espera encontrar una asociación estadísticamente significativa entre EN y desempeño cognitivo, con resultados a favor de AM con adecuado EN.

Palabras clave: disfunción cognitiva, envejecimiento, estado nutricional.

Keywords: Cognitive Dysfunction; Aging; Nutritional Status. 
Abstract ID: 188

\title{
EVALUACIÓN DE LAS PRÁCTICAS DE ALIMENTACIÓN Y FACTORES RELACIONADOS A LA RECUPERACIÓN NUTRICIONAL DE MENORES CON DESNUTRICIÓN AGUDA MODERADA Y SEVERA ATENDIDOS EN INSTITUCIONES PRESTADORAS DE SERVICIOS DE SALUD Y CENTROS DE RECUPERACIÓN NUTRICIONAL EN COLOMBIA
}

\author{
EVALUATION OF FEEDING PRACTICES AND RELATED FACTORS TO THE NUTRITIONAL RECOVERY IN INFANTS WITH MODERATE AND \\ SEVERE ACUTE MALNUTRITION ATTENDED IN HEALTH SERVICE INSTITUTIONS AND NUTRITIONAL RECOVERY CENTERS IN COLOMBIA
}

Yenny Paola Rueda Guevara', Omaira Valencia Estupiñán'.

Introducción: La prevalencia de desnutrición aguda en Colombia (1,6\%) en 2015 fue significativamente mayor a la reportada en 2010 (0,9\%); la cual tiene mayor prevalencia en menores de dos años. Pese a la importancia de la práctica de la lactancia materna (LM), se presentan bajas proporciones de lactancia materna exclusiva $(36,1 \%)$ y continua $(45,5 \%)$; y escasa evidencia de procesos exitosos de recuperación de esta práctica.

Objetivo: Evaluar las prácticas de LM, relactancia, alimentación complementaria y factores relacionados a la recuperación en menores de 24 meses con desnutrición aguda atendidos en tres Instituciones Prestadoras de Servicios de Salud (IPS) y trece Centros de Recuperación Nutricional (CRN).

${ }^{1}$ Fundación Santa Fe de Bogotá. Bogotá, Colombia.
Métodos: Investigación aplicada, descriptiva observacional mixta con componente cuantitativo y cualitativo. Se realizarán descriptivos con información cuantitativa a partir de fuentes secundarias como historias de atención. Se realizará observación participante y entrevistas semi estructuradas a profesionales y madres de los menores.

Resultados esperados: Evidenciar cambios en procesos de atención, en prácticas de alimentación de los menores, casos éxitos de relactancia y otros factores relacionados con la recuperación nutricional.

Palabras clave: lactancia materna, desnutrición, relactancia.

Keywords: Breastfeeding; Malnutrition; Relactation.

Abstract ID: 195

\section{RELACIÓN ENTRE MARCADORES INFLAMATORIOS Y EL ESTADO NUTRICIONAL, EN PACIENTES CON ENFERMEDAD DE ALZHEIMER DE APARICIÓN TEMPRANA (ANTIOQUIA-COLOMBIA)}

\author{
RELATIONSHIP BETWEEN INFLAMMATORY MARKERS AND NUTRITIONAL STATUS IN PATIENTS WITH EARLY-ONSET ALZHEIMER'S DISEASE \\ (ANTIOQUIA- COLOMBIA)
}

Gloria Cecilia Deossa Restrepo'.

Introducción: Los pacientes con demencia tipo Alzheimer de aparición temprana, presentan riesgo de deterioro del estado nutricional por factores alimentarios, socioeconómicos y aparición de marcadores inflamatorios relacionados con la enfermedad, sumado a la progresión acelerada de esta.

Objetivo: Establecer la relación entre los marcadores inflamatorios y el estado nutricional en pacientes con Enfermedad de Alzheimer de aparición temprana del Grupo de Neurociencias de Antioquia.

'Universidad de Antioquia. Medellín, Colombia.
Materiales y métodos: Estudio descriptivo transversal, cada paciente será evaluado para obtener información general, de salud, composición corporal, alimentaria, aspectos socioeconómicos, signos y síntomas clínicos, realización de marcadores bioquímicos (medición de interleuquina 6, proteína PCR, hemoleucograma, pre albúmina).

Palabras clave: enfermedad de Alzheimer temprano, evolución clínica, estado nutricional, consumo de alimentos, marcadores inflamatorios.

Keywords: Early Alzheimer's Disease; Clinical Evolution; Nutritional Status; Food Consumption, Inflammatory Markers.

Abstract ID: 196

\section{ENTRENAMIENTO FÍSICO Y LA RESPUESTA AL TRATAMIENTO DE INDUCCIÓN EN ADULTOS CON LEUCEMIA LINFOBLÁSTICA AGUDA}

\author{
PHYSICAL TRAINING AND THE RESPONSE TO INDUCTION THERAPY ON ADULTS WITH ACUTE LYMPHOBLASTIC LEUKEMIA \\ Christian Omar Ramos Peñafiel', Adán Germán Gallardo Rodríguez², Vanessa Fuchs Tarlovsky³, Mónica Patricia Bejarano Rosales , Karolina Álvarez-Altamirano 5 .
}

\begin{abstract}
Introducción: Los pacientes con leucemia linfoblástica aguda (LLA) tienen alto riesgo de desarrollar alteraciones en la composición corporal, mismas que deterioran la fuerza, movilidad, desempeño físico, calidad de vida y respuesta al tratamiento. A pesar de que el tratamiento farmacológico por si solo ayuda a la recuperación global de los pacientes, también se ha observado que realizar ejercicio durante la quimioterapia mejora la supervivencia a 5 años, calidad de vida y desempeño físico. Esta mejoría se ha asociado a la disminución de citocinas proinflamatorias séricas como la IL-15. Se ha demostrado que el ejercicio de resistencia es seguro en estos pacientes; sin embargo, el entrenamiento cruzado podría ofrecer mayores beneficios ya que no sólo se enfoca en aumentar la fuerza muscular en zonas específicas del cuerpo, sino que se centra en el fortalecimiento corporal global y en la mejoría de la movilidad articular, la estabilidad corporal y la resistencia aeróbica.

Objetivo: Este estudio tiene por objetivo evaluar el efecto de una rutina de entrenamiento cruzado en pacientes adultos jóvenes con LLA tipo B sobre la
\end{abstract}

respuesta al tratamiento de inducción, concentración sérica de IL-15, fuerza, desempeño físico y calidad de vida durante su estancia hospitalaria, en comparación con dos grupos control: entrenamiento de resistencia y recomendaciones generales de actividad física.

Métodos: Se realizará un ensayo clínico controlado ciego con 38 pacientes por grupo a quienes se les administrarán, durante 12 semanas (3 ciclos de tratamiento antineoplásico), el esquema de quimioterapia, requerimientos nutricionales específicos, y una rutina de ejercicios personalizados correspondientes el grupo incluido.

Palabras clave: entrenamiento, leucemia linfoblástica aguda, interleucina 15, composición corporal, desempeño físico.

Keywords: Physical Training; Acute Lymphoblastic Leukemia; Interleukin-15; Body Composition; Physical Performance. 
'Hematología, Investigador en Ciencias, Adscrito Servicio de Hematología, Hospital General de México “Dr. Eduardo Liceaga”, México. ${ }^{2}$ Nutrición Clínica, Hospital General de México “Dr. Eduardo Liceaga”, México.

${ }^{3}$ Nutrición Clínica; Investigadora en Ciencias; jefe del Servicio Nutrición Clínica, Hospital General de México "Dr. Eduardo Liceaga", México.

"Nutrición Clínica; Investigador en Ciencias; Técnico en Nutrición, Hospital General de México “Dr. Eduardo Liceaga”, México.

${ }^{5}$ Nutrición Clínica; Investigador en Ciencias B., Hospital General de México “Dr. Eduardo Liceaga”, México.

\author{
Abstract ID: 198
}

\title{
PRESENCIA DE FTALATOS EN LA ALIMENTACIÓN INFANTIL: UNA REVISIÓN DE LA LITERATURA
}

\section{APPEARANCE OF PHTHALATES IN INFANT FOOD: A REVIEW OF THE LITERATURE}

Angelica Johana Martínez Bravo'.

Introducción: Los materiales empleados en los empaques de alimentos, principalmente plásticos y resinas, están en contacto estrecho con los mismos. Los plastificantes, tienen como función mejorar la flexibilidad y la manipulación durante el procesado de los polímeros. Entre los plastificantes empleados cabe destacar los ftalatos, continúan siendo la clase más importante, representando el $65 \%$ del consumo mundial de este tipo de aditivos en la industria de los plásticos (5). Los ftalatos son contaminantes universales presentes en la sociedad actual, que son usados en juguetes, empaques de alimentos, pinturas, materiales para construcción, entre otros. Entre los ftalatos y derivados de los mismos hay sustancias que han sido asociadas a diferentes problemas de salud como disrupción endocrina y tienen implicaciones en la salud, nutrición y alimentación infantil.

'Universidad Nacional de Colombia. Bogotá, Colombia.
Objetivos: Realizar una revisión sistemática de literatura que permita identificar, extraer y recopilar la información relevante acerca de la presencia de Ftalatos en la alimentación infantil, sus riesgos y potenciales efectos nocivos.

Métodos: El presente protocolo de investigación se soporta en el protocolo PRISMA-P (Preferred Reporting Items for Systematic Reviews and Meta-Analyses), cumplimiendo con lista de verificación de 17 elementos, que garantizará una sólida revisión, replicación y verificación de la evidencia obtenida.

Resultados esperados: Generar una actualización, que incluya las implicaciones para la Práctica en Nutrición Clínica y Toxicología, las recomendaciones para la investigación futura y la toma de decisiones en salud pública y nutrición clínica.

Palabras clave (DeCS): nutrición del niño, alimentos infantiles, nutrición del adolescente, toxicidad, embalaje de alimentos, inocuidad de los alimentos.

Keywords (MeSH): Infant Food; Child Nutrition Sciences; Toxicity; Food Safety; Food Packaging. 